\title{
ESTUDO DO COMPORTAMENTO DO BANHO NO CONVERTEDOR LD COM SOPRO COMBINADO*
}

\author{
Breno Totti Maia ${ }^{1}$ \\ Rafael Kajimoto Imagawa² \\ Roberto Parreiras Tavares ${ }^{3}$
}

\section{Resumo}

O BOF, Basic Oxygen Furnace, ou convertedor LD, é o processo mais utilizado industrialmente para o refino primário do ferro gusa atualmente. Para aumentar a produtividade do BOF deve-se diminuir o tempo de cada ciclo de operação, especialmente o tempo de sopro aumentado a taxa de descarburação. Durante o sopro do convertedor existem três etapas. A segunda etapa do sopro a taxa de descarburação é alta e constante sendo o fator limitante é a disponibilidade do oxigênio no banho e determina o tempo de sopro. Assim para aumentar a descarburação pode-se melhorar a interação do jato de oxigênio com o banho, configurando o convertedor. Estudando o sopro combinado em um modelo fisíco de um convertedor LD de 220t, foi testado diferentes bicos de lança com um sopro de $160 \mathrm{Nm}^{3} / \mathrm{h}$ de vazão e vazões de $4 \mathrm{Nm}^{3} / \mathrm{h}$ e $6 \mathrm{Nm}^{3} / \mathrm{h}$ nas ventaneiras. Através dos testes pode-se observar que aumentando o número de furos no bico o jato teve menor penetração, maior área de agitação e as projeções tiveram menor altitude, já a injeção pelas ventaneiras aumentou a penetração do jato e diminuiu as projeções. Foi calculado também valores para a constante $\mathrm{K}$ para cada configuração, assim é possível prever a penetração do jato industrialmente.

Palavras-chave: BOF; Sopro; Bottom blowing; Cold model.

\section{STUDY BATH BEHAVIOR IN LD CONVERTER WITH BOTTOM BLOWING}

\begin{abstract}
The BOF, Basic Oxygen Furnace or LD converter, is the process most widely used industrially for primary refining of pig iron today. To increase the productivity of BOF you should decrease the time of each cycle of operation, especially the blowing time. To decrease the BOF blowing time, should have a higher decarburization rate. During the blowing operation of the converter it is known that there are three stages. In the blow second stage the decarburization rate is high and constant, the limiting factor is the availability of oxygen in the bath. Thus to increase the decarburization can improve the interaction of oxygen jet with the bath. Studying the combined blow on a physical model of a LD converter 220T, has been tested different lance nozzles with a blow of $160 \mathrm{Nm} 3 / \mathrm{h}$ and flow of $4 \mathrm{Nm} 3 / \mathrm{h}$ and $6 \mathrm{Nm} 3 / \mathrm{h}$ in the tuyeres. Through the tests it can be seen that increasing the number of holes in the jet nozzle, it had less penetration and the projections had a lower elevation, as by injection tuyeres effect, it increased penetration of the jet and decreased projections. It was also calculated values for the constant $\mathrm{K}$ for each configuration, so it is possible to predict the penetration of jet industrially.
\end{abstract}

Keywords: BOF; Top Blow; Bottom Blown; Cold Model.

Sócio da ABM; Engenheiro Metalurgista e Doutor Pesquisador da LUMAR METALS;

Aluno de Graduação do Curso de Engenharia Metalúrgica da UFMG, Belo Horizonte, MG, Brasil.

Sócio da ABM; Engenheiro Metalurgista; Professor Doutor do Departamento de Engenharia Metalúrgica da UFMG, Belo Horizonte, MG, Brasil. 


\section{INTRODUÇÃO}

O convertedor LD é o mais utilizado industrialmente. Este processo consiste em soprar oxigênio no banho metálico e assim o carbono presente no banho reage com o oxigênio formando $\mathrm{CO}$ e $\mathrm{CO}_{2}$, diminuindo o teor de carbono do banho. Existem três tipos de operação para o convertedor LD, sendo elas:

- Sopro de oxigênio e gás inerte pelo fundo do vaso utilizando ventaneiras submersas no banho;

- Sopro de oxigênio pelo topo utilizando uma lança;

- Sopro combinado, onde há o sopro de oxigênio pelo topo e de gás inerte pelas ventaneiras no fundo.

A Figura 1 apresenta um desenho esquemático de sopro combinado em duas vazões de borbulhamento.
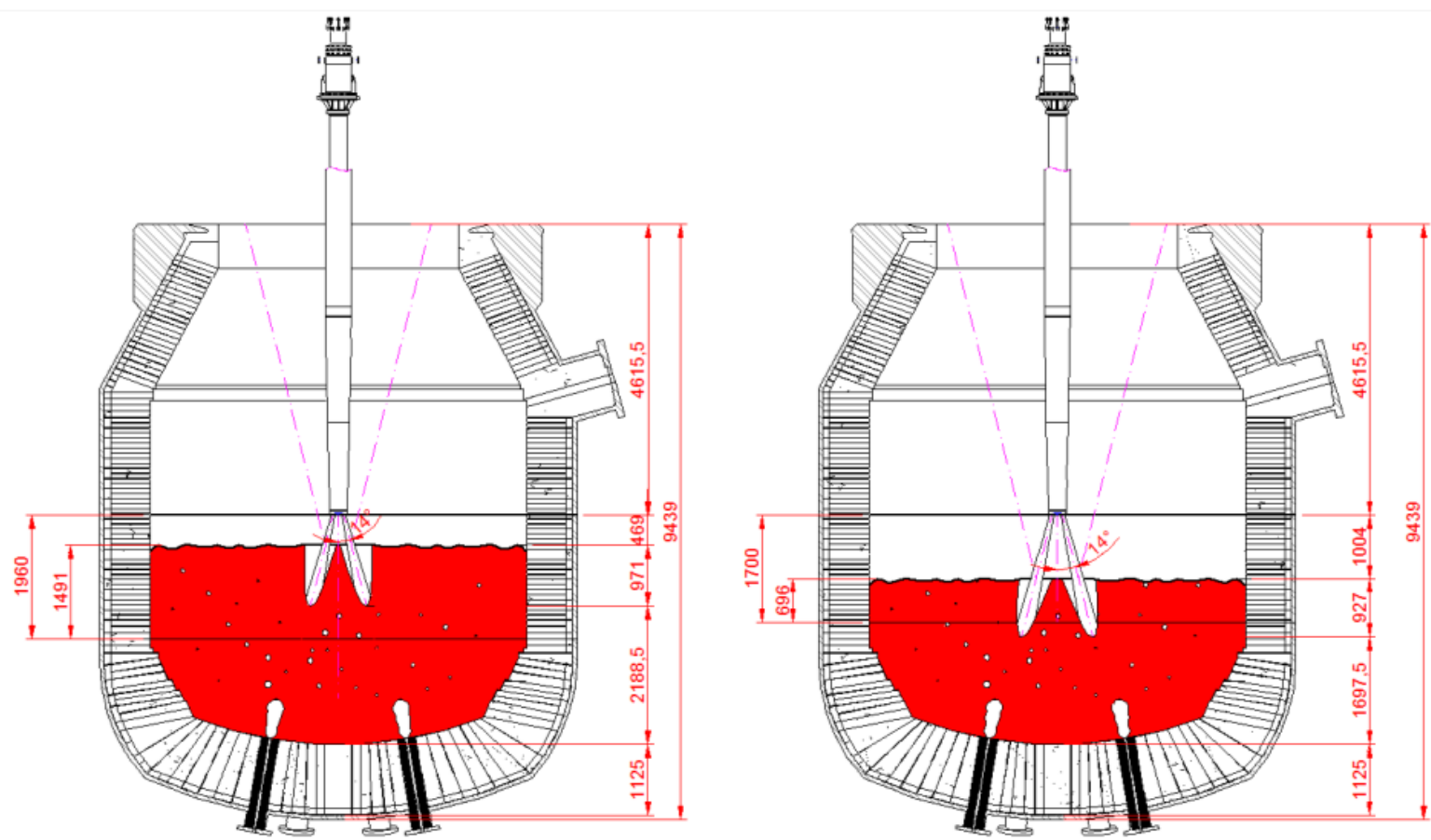

Figura 1 - Desenho esquemático de sopro combinado em vazões de borbulhamento variadas: a) elevada e b) baixa (Cedido por Lumar Metals).

A etapa da operação do convertedor LD que tem a maior duração, responsável por aproximadamente $60 \%$ do tempo total de uma corrida, é o sopro. Com isso, para reduzir o tempo de ciclo e aumentar a produtividade, deve-se diminuir o tempo de sopro. Para diminuir o tempo de sopro deve-se aumentar a taxa de descarburação, que é afetada pelas diferentes configurações do convertedor, como a vazão de sopro, altura da lança, tipo de sopro (sopro simples, combinado), tipo de bico da lança e vazão das ventaneiras.Com o objetivo de analisar como alguns destes fatores afetam o jato de oxigênio, sua interação com o banho metálico e com isso o seu efeito no tempo de mistura do processo, este trabalho foi conduzido. 


\section{MATERIAIS E MÉTODOS}

\subsection{Modelagem Física}

Para a realização da simulação do convertedor LD foi utilizado um modelo de acrílico em escala 1/8 de um convertedor de 220t. As dimensões do modelo são apresentadas na Figura 2.

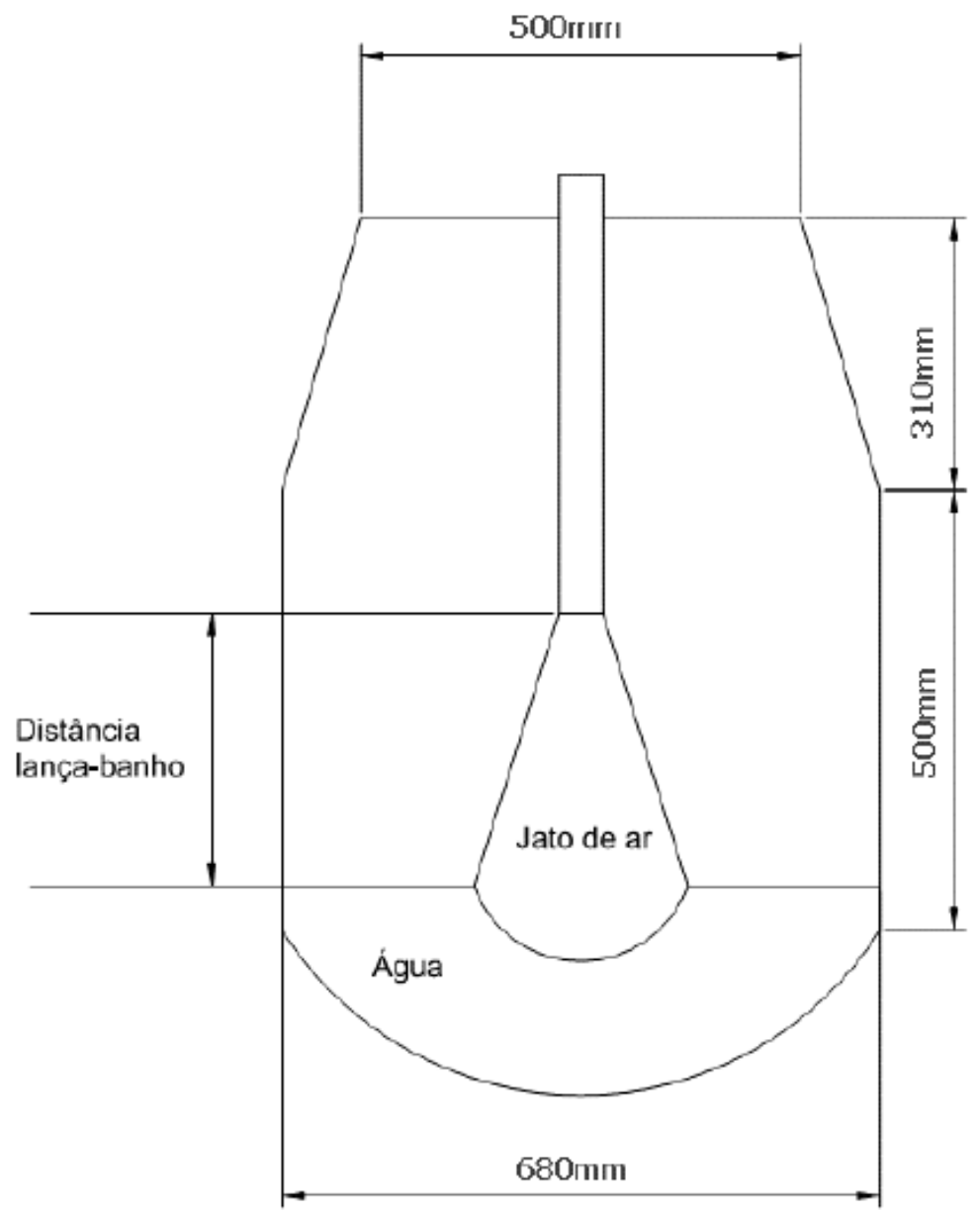

Figura 2 - Dimensões do modelo físico do convertedor (Maia, 2013).

$\mathrm{Na}$ simulação do sopro da lança e das ventaneiras, foi utilizado um compressor de $22,5 \mathrm{~kW}$ com uma vazão máxima de $189 \mathrm{~m}^{3} / \mathrm{h}$. Para controlar a vazão do jato da lança foram utilizados registros em série e um medidor de vazão, já para o controle da vazão das ventaneiras também foram utilizados dois registros, um medidor de vazão e um rotâmetro para cada ventaneira. Os dois registros em série foram utilizados em ambos os casos, pois era necessário atingir rapidamente a vazão desejada para cada teste, pois o banho fica turvo rapidamente, impedindo a visualização da penetração do jato. A distância lança-banho utilizada foi de $380 \mathrm{~mm}$, simulando a altura utilizada em algumas indústrias e a configuração das ventaneiras utilizada foi a sugerida por Lima (2011).

\subsection{Configuração dos bicos}

As configurações de bicos utilizados neste estudo foram 3,4,5 e 6 furos com ângulos de $8,10,15$ e $17,5^{\circ}$. A geometria interna do bocal é constituída de uma parábola, 
formando um trecho convergente em seguida um segmento reto curto, onde se encontra o diâmetro crítico e ao fim um trecho divergente, como é detalhado na Figura 3.

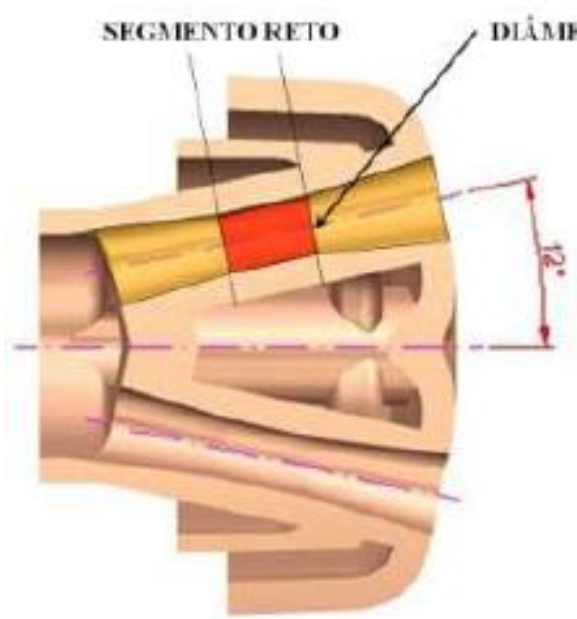

(a)

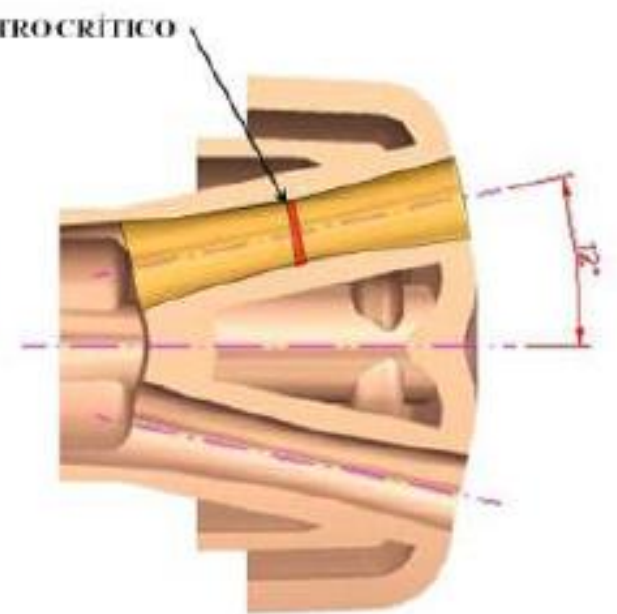

(c)

Figura 3 - Geometria interna do bocal (Maia, 2013).

\subsection{Comparações do modelo físico com o industrial}

Para analisar a semelhança entre o processo industrial e o modelo físico foram comparadas algumas características fundamentais do processo e dos fluidos envolvidos. Os dados analisados estão dispostos na Tabela I.

Tabela I - Números adimensionais

\begin{tabular}{|c|l|c|c|}
\hline & & Industrial & Modelo \\
\hline $\mathrm{Fr}^{*}$ & Froude Modificado & 0,089 & 0,089 \\
\hline $\mathrm{Re}^{*}$ & Reynolds Modificado & $5,588^{*} 10^{5}$ & $1,014^{*} 10^{5}$ \\
\hline $\mathrm{We}^{*}$ & Weber Modificado & $1,731^{*} 10^{4}$ & $1,395^{*} 10^{3}$ \\
\hline
\end{tabular}

Na Tabela I são apresentados os mesmos números adimensionais utilizados por Meidani et al. (2004) e Diaz-Cruz et al. (2002) em trabalhos semelhantes.

\subsection{Procedimentos}

Para realização dos experimentos, o nível de aço é representado com água até a altura de $250 \mathrm{~mm}$ do fundo do vaso. As ventaneiras com vazão de $4 \mathrm{~m}^{3} / \mathrm{h}$ ou $6 \mathrm{~m}^{3} / \mathrm{h}$. Com a vazão ajustada no primeiro registro, fecha-se o segundo registro e espera-se até o banho estabilizar, quando o banho estiver imóvel liga-se a câmera filmadora e inicia-se o teste, abrindo rapidamente o registro das ventaneiras e em seguida o da lança. Para a filmagem foi utilizado uma câmera e um refletor, eles foram posicionados em lados opostos ao modelo do convertedor, ou seja, o refletor é direcionado para a câmera atrás do modelo físico. Com está configuração conseguiu-se um maior contraste entre o a parte do banho que foi atingida pelo jato e a parte que não foi afetada. 


\subsection{Medição da penetração do jato}

Para medir a penetração foi utilizado o programa Adobe Premiere para a edição do vídeo, aumentando o contraste da imagem. Determinado o momento em que o jato é despachado, adiciona-se 3 segundos e extrai-se um quadro da filmagem. É comparado a profundidade do jato com a distância conhecida, utilizando o software ImageJ, que analisando quantos quadros correspondem aos $250 \mathrm{~mm}$, é possível calcular quantos milímetros correspondem à profundidade de penetração do jato, como indicado na Figura 4.

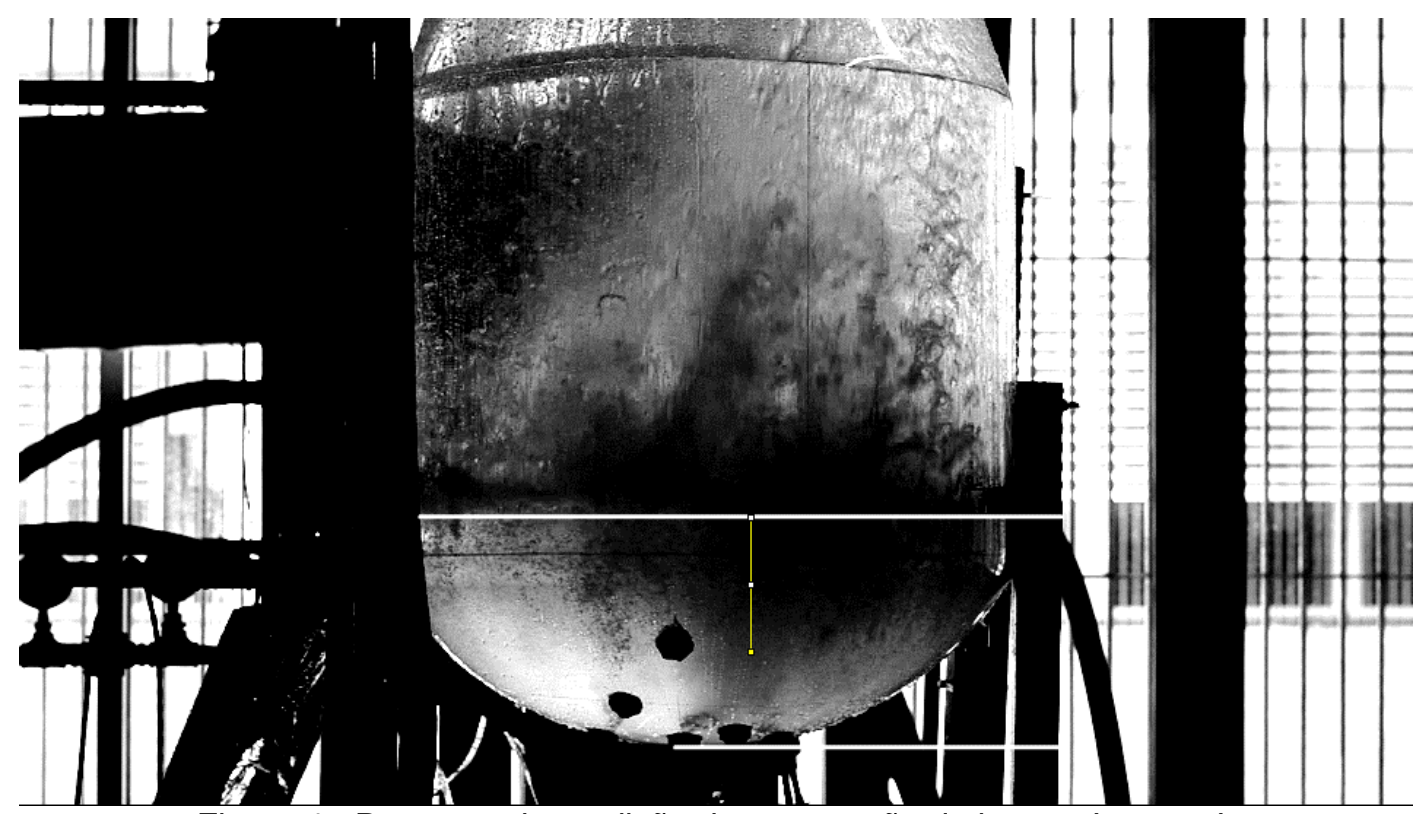

Figura 4 - Processo de medição da penetração do jato no ImagaeJ.

\section{RESULTADOS E DISCUSSÃO}

\section{a. Análise da influência do número de furos}

A Figura 5 apresenta sopro pelo topo é o comportamento da penetração de jato.

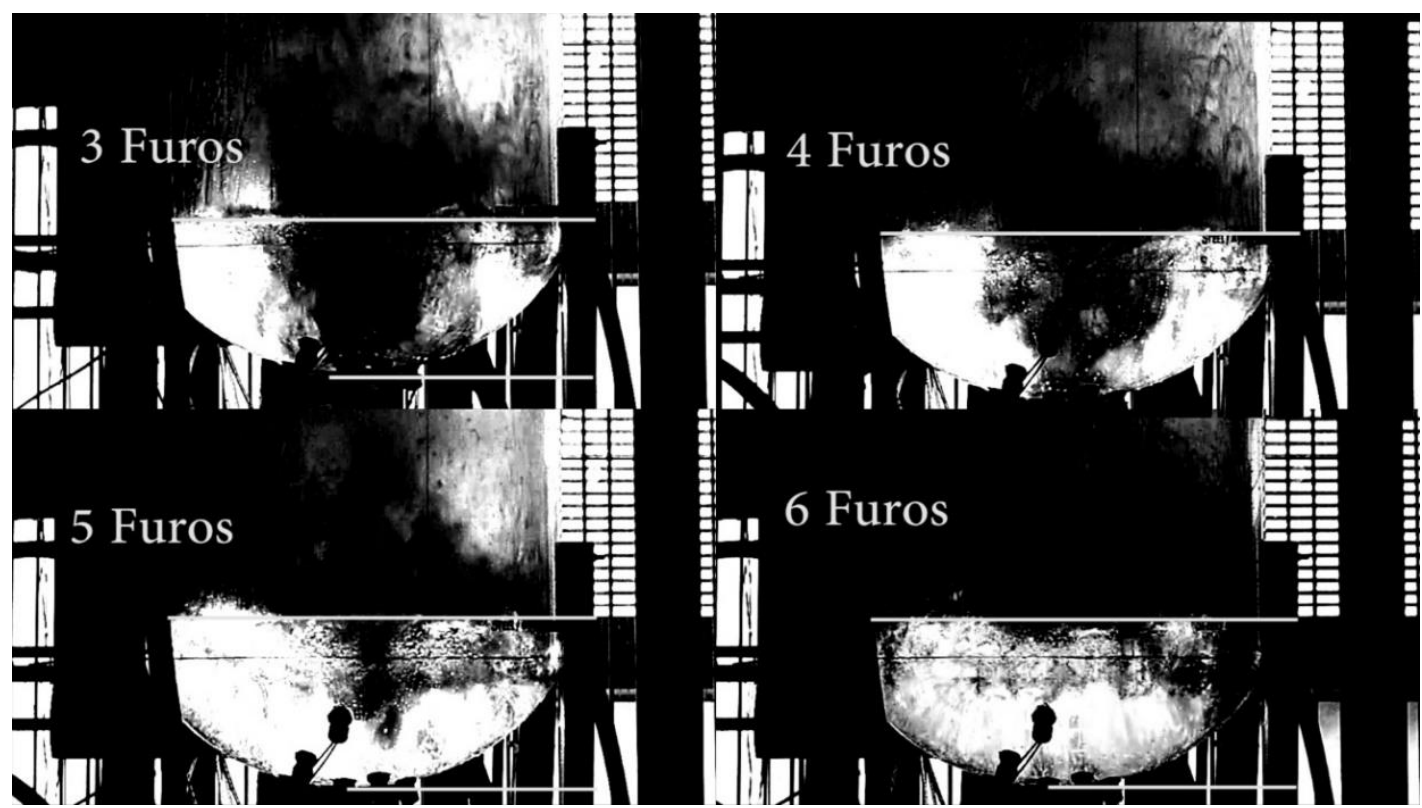

Figura 5- Sopro simples com vazão de $160 \mathrm{Nm}^{3} / \mathrm{h}$. 
O quadro da Figura 5 apresenta sopro pelo topo com uma vazão de $160 \mathrm{Nm}^{3} / \mathrm{h}$. Com aumento do número de furos do bico há uma redução na penetração do jato e aumento da área penetrada (dimple), caracterizando um sopro macio potencialmente bom para desfosforação. A redução do número de furos de sopro torna-o com forte penetração, utilizado para descarburar considerando a altura de lança de $380 \mathrm{~mm}$. Outro ponto que pode ser observado é que com o aumento do de furos do bico o splashing fica distribuído acima do nível de banho estático, reduzindo a incidência de projeções. No teste com o bico de três furos pode-se observar que a projeção ficou concentrada na região da lança e alcançou grande altura maior em relação aos demais. O splashing concentrado na região da lança e com um alcance de altura elevado pode formar cascão na lança, fazendo necessária a parada de processo para a limpeza e com isso a perda de tempo e produtividade.

É possível observar também que a vazão de $160 \mathrm{Nm}^{3} / \mathrm{h}$, utilizada no estudo combinado com o bico de 3 furos, promove um sopro muito duro, que apesar de aumentar a taxa de descarburação, atinge o fundo do vaso, o que pode reduzir a vida dos refratários. Também é possível observar que existem zonas mortas perto dos munhões do convertedor, que na terceira etapa de sopro, quando o fator limitante é a difusão do carbono, pode levar a uma menor taxa de descarburacão.

\section{b. Análise da influência das ventaneiras i. Penetração de Jato}

Comparando os testes com sopro combinado com o teste com sopro simples é possível observar que com a adição das ventaneiras a penetração do jato aumentou. Um dos motivos para isso pode ser a redução da tensão superficial do banho, gerada pela redução de densidade do banho. Os resultados de penetração do jato, mostrados na Tabela II e pelas Figura 5, Figura 6, Figura 7, indicam que com a injeção de jato pelas ventaneiras fez com que o jato tivesse maior penetração no banho, assim como o aumento da vazão das ventaneiras. É importante destacar que as medidas de penetração do jato foram realizadas por apenas um observador

Tabela II: Penetração do jato para os testes

\begin{tabular}{|c|c|c|c|c|}
\hline BicolVazão & 3 & 4 & 5 & 6 \\
\hline $0 \mathrm{Nm}^{3} / \mathrm{h}$ & $\mathbf{0 , 2 5 0}$ & $\mathbf{0 , 2 5 0}$ & $\mathbf{0 , 1 4 0}$ & $\mathbf{0 , 0 9 0}$ \\
\hline $4 \mathrm{Nm}^{3} / \mathrm{h}$ & $\mathbf{0 , 2 5 0}$ & $\mathbf{0 , 2 5 0}$ & $\mathbf{0 , 1 5 0}$ & $\mathbf{0 , 0 8 7}$ \\
\hline $6 \mathrm{Nm}^{3} / \mathrm{h}$ & $\mathbf{0 , 2 5 0}$ & $\mathbf{0 , 2 5 0}$ & $\mathbf{0 , 1 7 0}$ & $\mathbf{0 , 1 0 5}$ \\
\hline
\end{tabular}




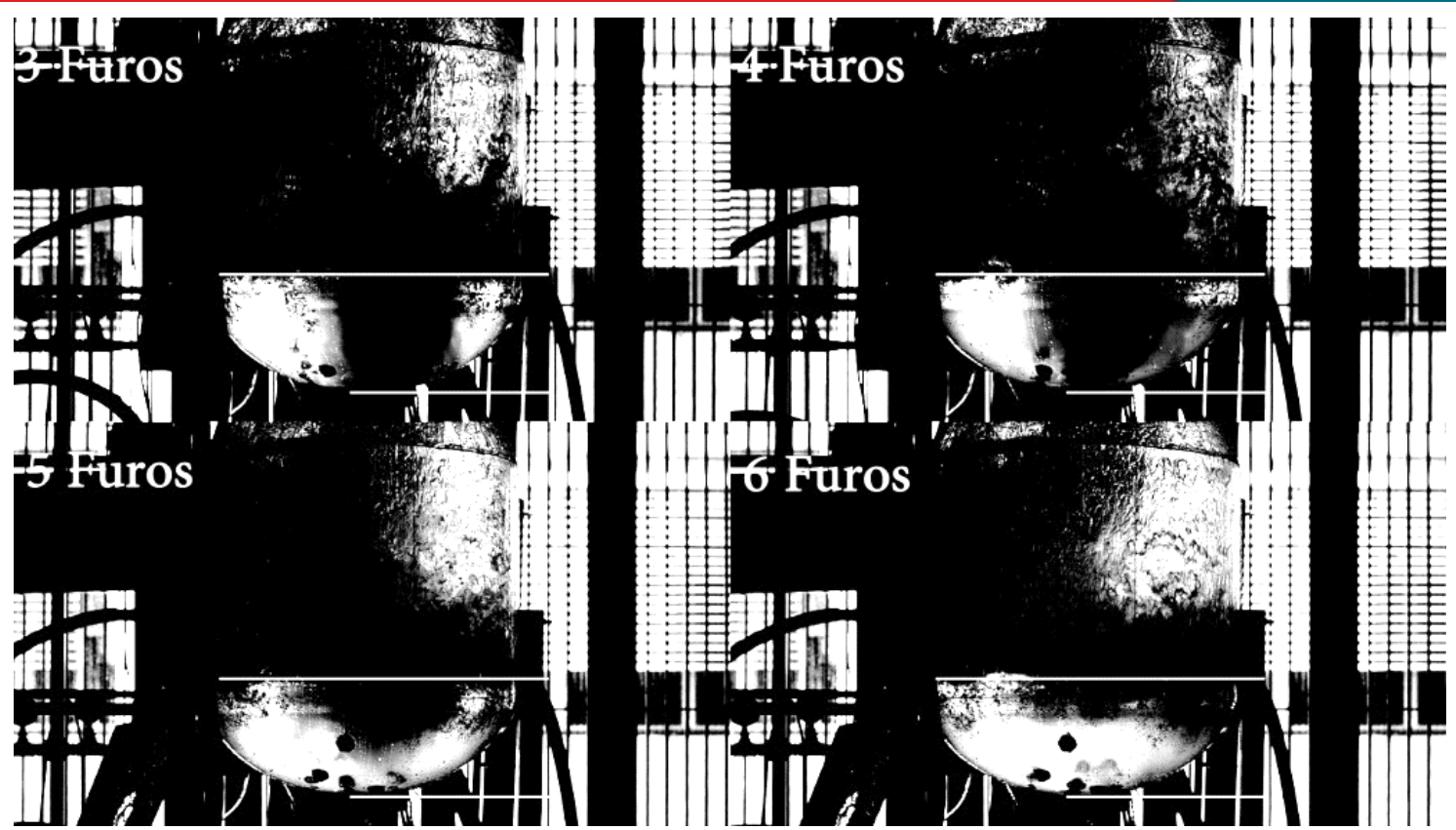

Figura 6: Testes com sopro combinado, lança com $160 \mathrm{Nm}^{3} / \mathrm{h}$ e ventaneiras com $4 \mathrm{Nm}^{3} / \mathrm{h}$.

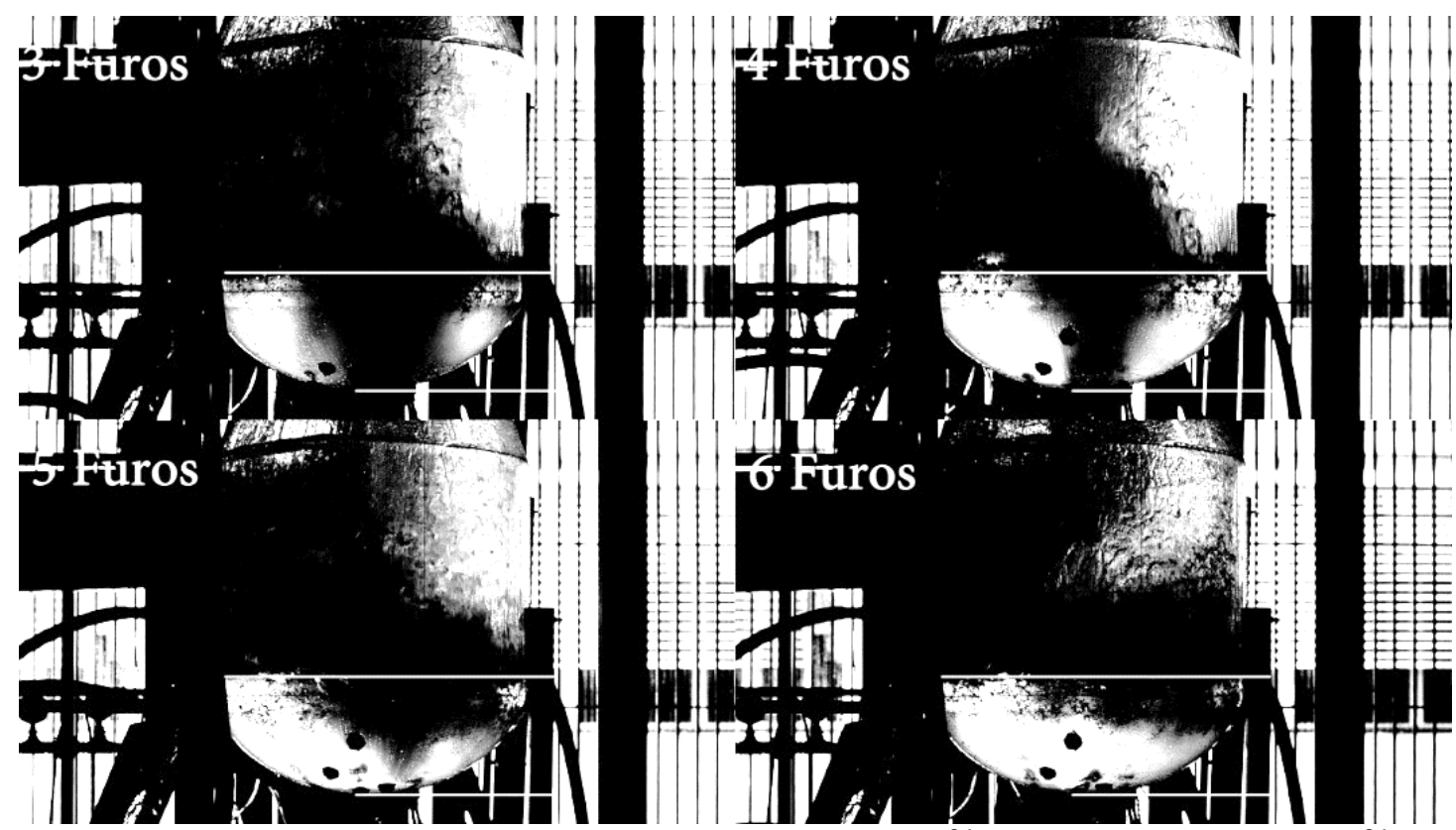

Figura 7: Testes com sopro combinado, lança com $160 \mathrm{Nm}^{3} / \mathrm{h}$ e ventaneiras com $6 \mathrm{Nm}^{3} / \mathrm{h}$

$\mathrm{Na}$ Figura 8 pode-se observar que variando do número de furos para diferentes vazões das ventaneiras a penetração do jato tem comportamento parecido. 


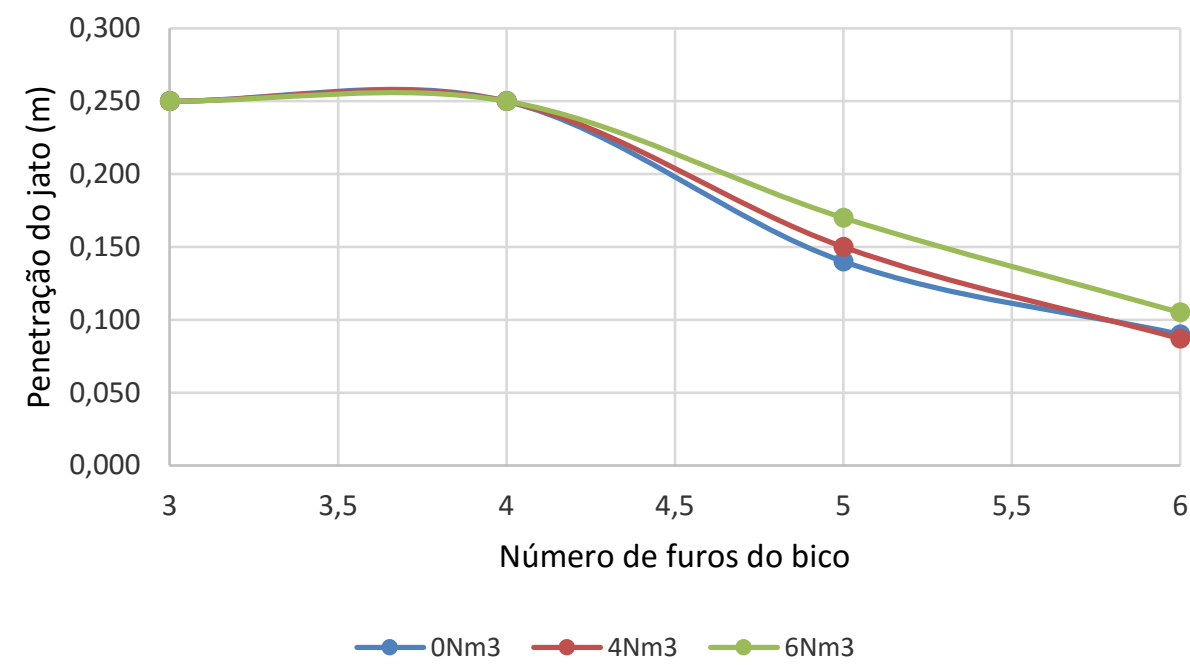

Figura 8: Gráfico de penetração do jato para bicos de 3,4,5 e 6 furos.

O aumento da penetração do jato em função do aumento da vazão das ventaneiras comprova o efeito de um dos parâmetros descrito por Meidani et al. (2004), a densidade do banho. Com o aumento da vazão das ventaneiras a densidade do banho reduz, como indicado na Tabela III, ou seja, quanto menor a densidade do banho maior a penetração do jato, sendo as outras configurações mantidas.

Tabela III: Densidade do banho

\begin{tabular}{|c|c|}
\hline $\begin{array}{c}\text { Vazão das ventaneiras } \\
\left(\mathrm{Nm}^{3} / \mathrm{h}\right)\end{array}$ & $\begin{array}{c}\text { Densidade do } \\
\text { banho }\left(\mathrm{kg} / \mathrm{m}^{3}\right)\end{array}$ \\
\hline 0 & 1000,0 \\
\hline 4 & 491,1 \\
\hline 6 & 391,8 \\
\hline
\end{tabular}

Nos valores calculados da Tabela III foram considerados os seguintes aspectos: a) o banho estático mesmo com borbulhamento considerado plano, b) Regime estacionário, ou seja, em uma unidade de tempo as bolhas ficam contidas e distribuídas no interior do banho. Devido essas considerações os valores de densidade calculadas são menores que as simuladas, mas foram usadas como referência para comparação com e sem ventaneiras.

\section{ii. Splashing}

Com a introdução das ventaneiras pode-se observar, comparando a Figura 9, Figura 10 e Figura 11 que há uma significante redução do splashing, esse efeito fica evidente para a lança de 6 furos pois ela possui uma projeção mais uniforme, como foi também observado no estudo de Miguel, Barrón e González (2007). Isso ocorre, pois o gás injetado pelas ventaneiras faz com que o slopping no banho fique menor e se desloque para as bordas do vaso diminuindo as projeções, porém como as projeções estão concentradas na parte cilíndrica do vaso pode causar um maior desgaste dos refratários nesta área. 


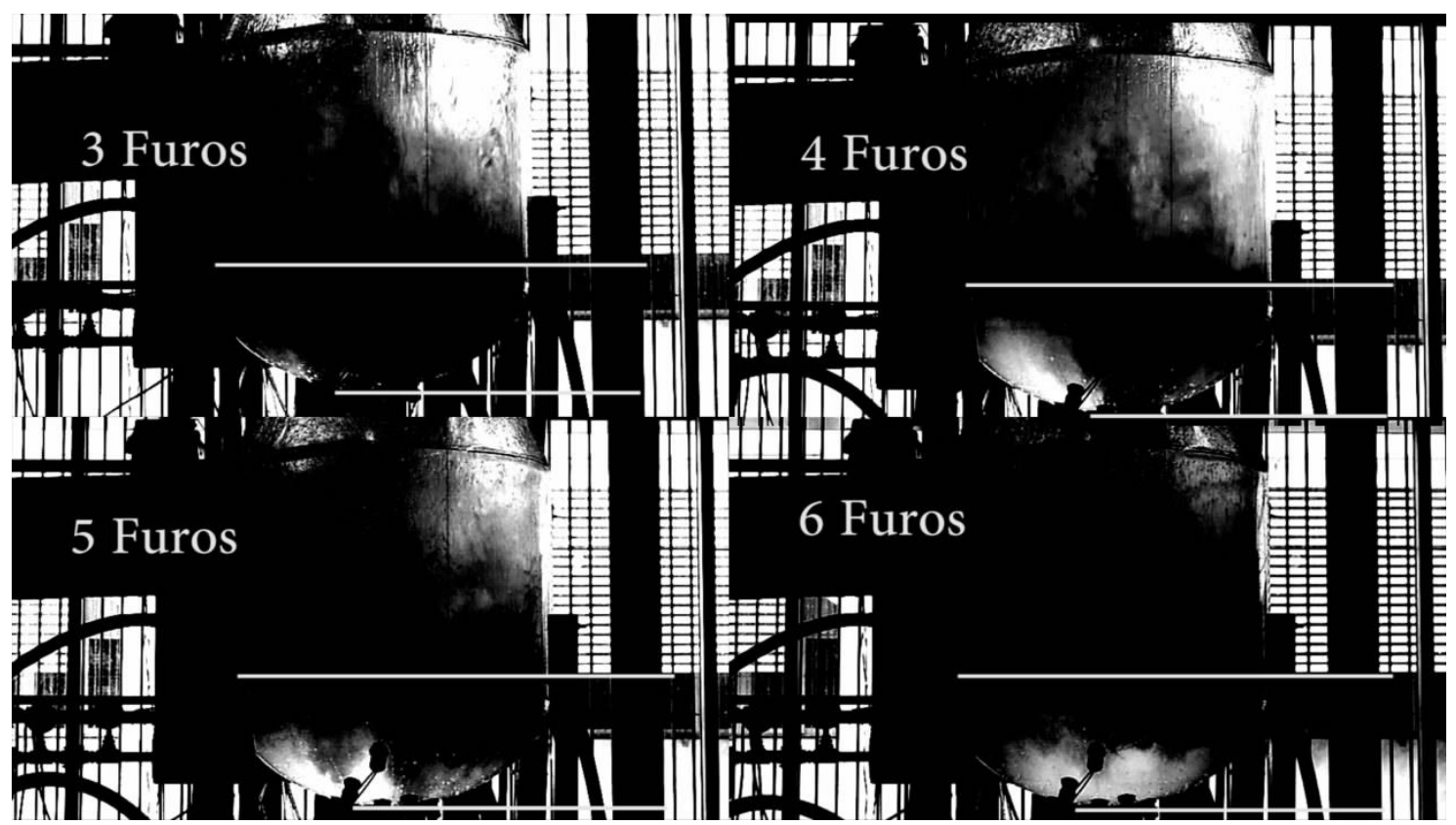

Figura 9: Splashing para o sopro simples (DBL 0,38m Vazão $160 \mathrm{Nm}^{3} / \mathrm{h}$ ).

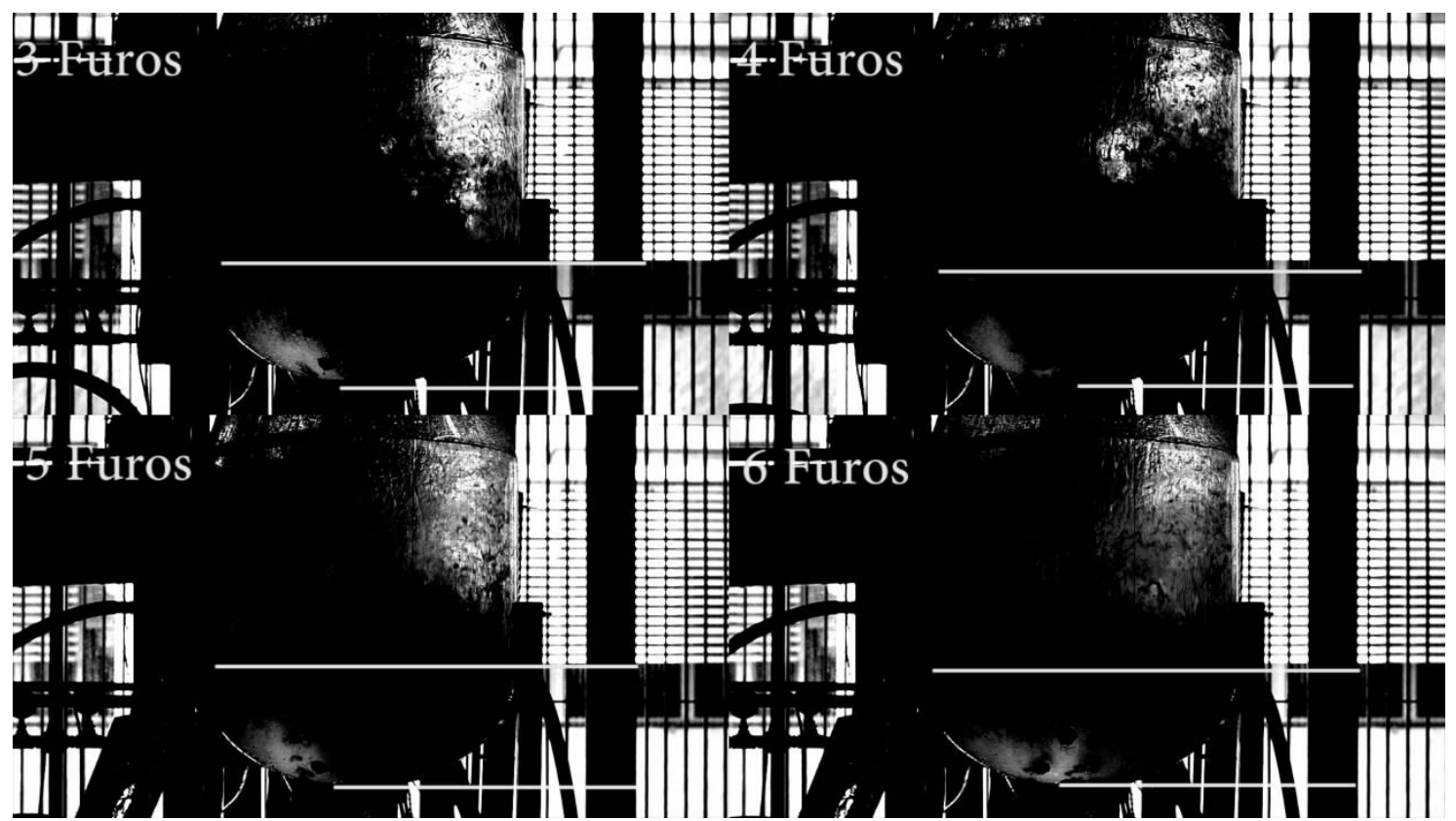

Figura 10: Splashing para o sopro combinado (DBL $0,38 \mathrm{~m}$ Vazão - $160 \mathrm{Nm}^{3} / \mathrm{h}$, Ventaneiras - $4 \mathrm{Nm}^{3} / \mathrm{h}$ ) 


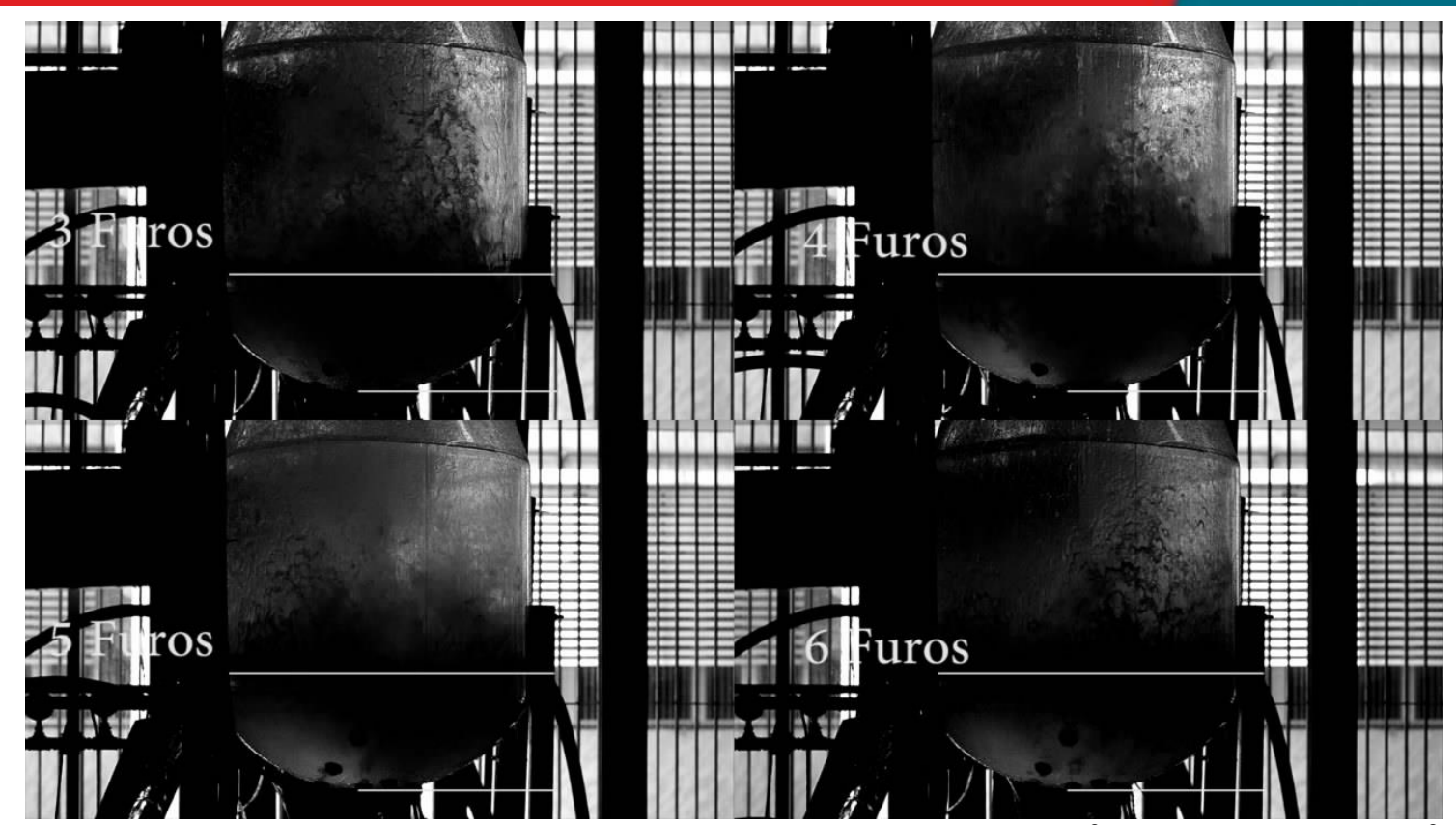

Figura 11: Splashing para o sopro combinado (DBL 0,38m Vazão - $160 \mathrm{Nm}^{3} / \mathrm{h}$, Ventaneiras - $6 \mathrm{Nm}^{3} / \mathrm{h}$ ).

\subsection{Cálculos dos valores da constante $\mathrm{K}$}

Para o calculo de $\mathrm{K}$ utilizou-se a equação 3.1 desenvolvida por Maia (2013).

$\frac{\pi \cdot \rho_{g} \cdot V_{s}^{2} \cdot D_{s}^{2} \cdot \cos \theta \cdot n}{4 \cdot \rho_{l} \cdot g \cdot D B L}=\frac{2}{K^{2}} \cdot \frac{P_{j}}{D B L} \cdot\left(1+\frac{P_{j}}{D B L+\cos \theta}\right)^{2}$

Onde: $\rho_{g}$ - Densidade do gás, $\rho$ - Densidade do líquido, $V_{s}-$ Velocidade do jato na saída do bocal, $D_{s}$ - Diâmetro da saída do bocal, $n$ - Número de furos, $\theta$ - Ângulo do bocal, Pj - Penetração do jato, DBL - Distância Lança Banho, g - Gravidade.

Os resultados destes cálculos estão na Tabela IV.

Tabela IV: Valor de K para os testes.

\begin{tabular}{|c|c|c|c|c|}
\hline & \multicolumn{4}{|c|}{ Número de furos do bico de lança } \\
\hline Vazão Ventaneira & 3 & 4 & 5 & 6 \\
\hline $0 \mathrm{Nm}^{3}$ & $\mathbf{6 , 9 2}$ & $\mathbf{7 , 0 1}$ & $\mathbf{4 , 3 5}$ & $\mathbf{3 , 1 8}$ \\
\hline $4 \mathrm{Nm}^{3}$ & $\mathbf{4 , 8 5}$ & $\mathbf{4 , 9 2}$ & $\mathbf{3 , 2 2}$ & $\mathbf{2 , 3 5}$ \\
\hline $6 \mathrm{Nm}^{3}$ & $\mathbf{4 , 3 3}$ & $\mathbf{4 , 3 9}$ & $\mathbf{3 , 1 8}$ & $\mathbf{2 , 2 2}$ \\
\hline
\end{tabular}

Pode-se observar que aumentando o número de furos diminui-se o $\mathrm{K}$, o que já era esperado, pois é uma reposta ao aumento do ângulo dos furos da lança. Com o aumento da vazão das ventaneiras também há uma redução do $\mathrm{K}$, respondendo a redução de densidade do banho. $\mathrm{O}$ fator $\mathrm{K}$ mede resistências de trajeto e banho a penetração de jato desde a saída do bocal supersônico.

$\mathrm{Na}$ Figura 12 pode-se observar que $\mathrm{o}$ valor de $\mathrm{K}$ para as três vazões das ventaneiras tem um comportamento similar. Os bicos de 3 e 4 furos também têm valor similar de $\mathrm{K}$ no experimento pois nos dois casos a penetração do jato tocou 0 fundo do vaso. O efeito combinado do aumento da vazão de ventaneiras e aumento de número de furos do bico levam ao menor valor de K. Esse resultado pode ser interpretado com um aumento da movimentação do banho, podendo ser benéfico a homogeneização da corrida e redução do tempo de sopro. 

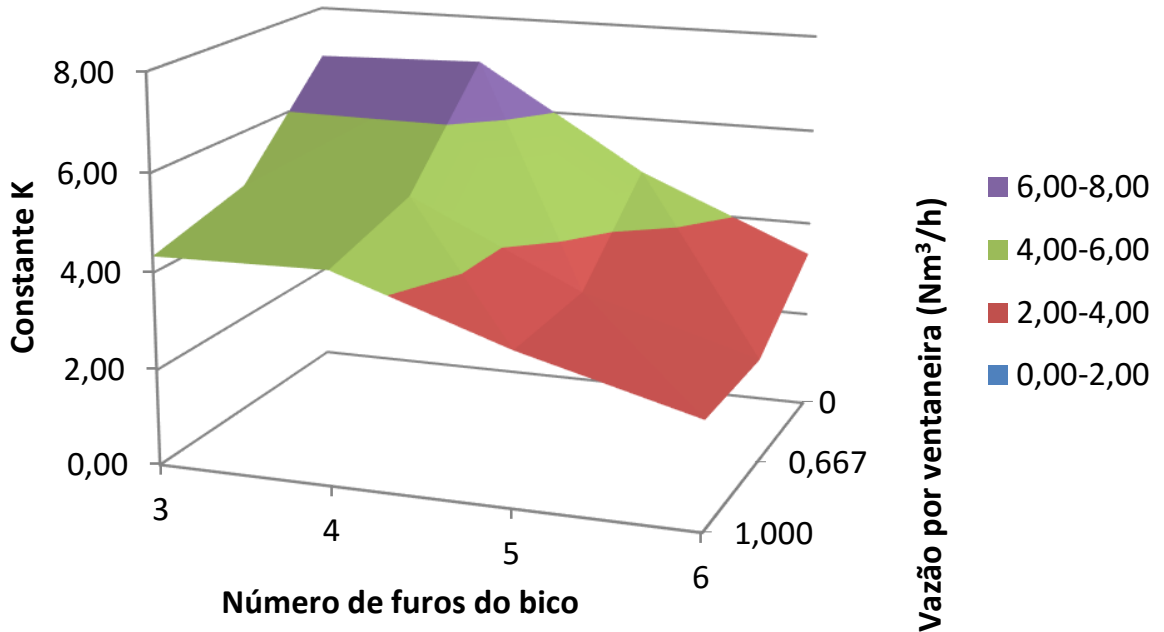

Figura 12: Valor de $\mathrm{K}$ em função do número de furos e da vazão de cada ventaneira.

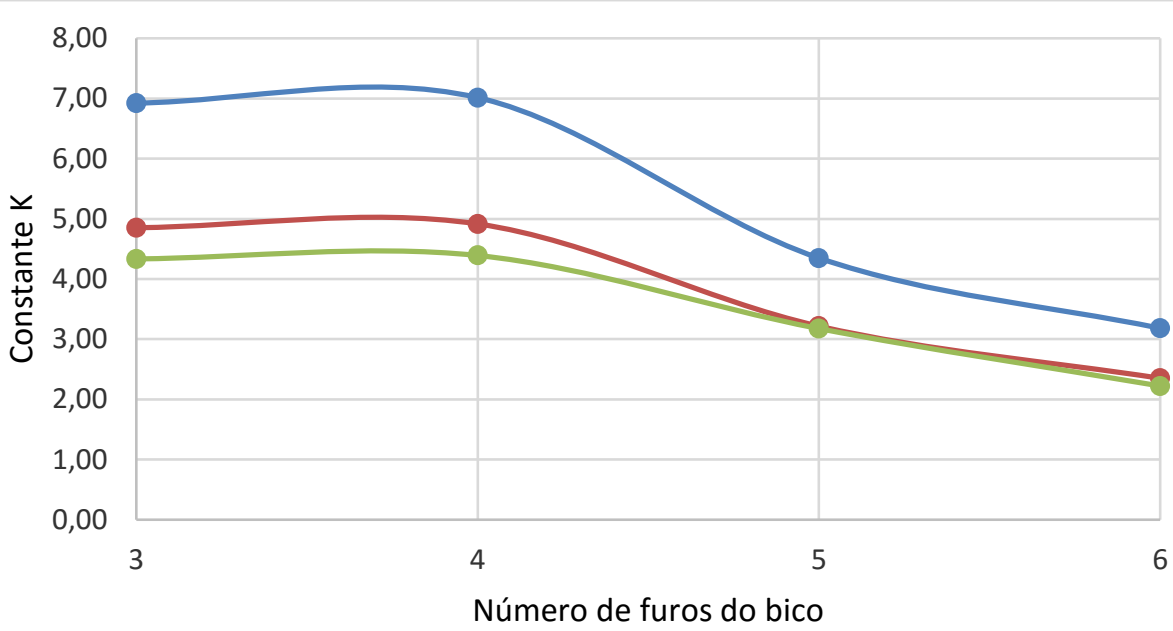

$\longrightarrow 0 \mathrm{Nm} 3 \longrightarrow 4 \mathrm{Nm} 3 \longrightarrow 6 \mathrm{Nm} 3$

Figura 13 - Gráfico da constante K para os bicos de 3,4,5 e 6 furos.

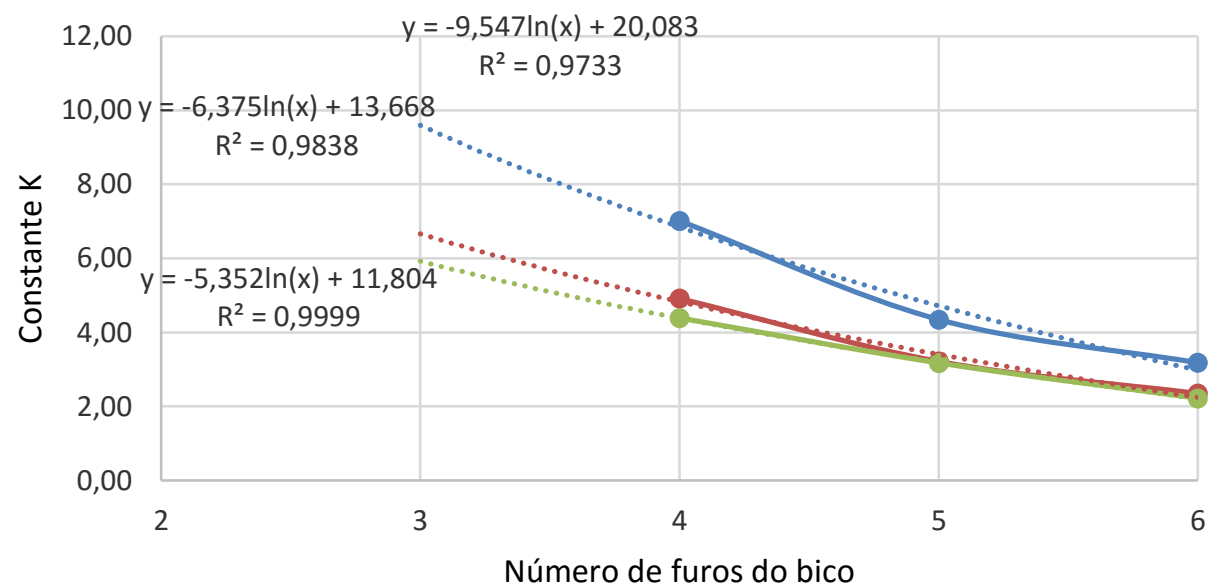

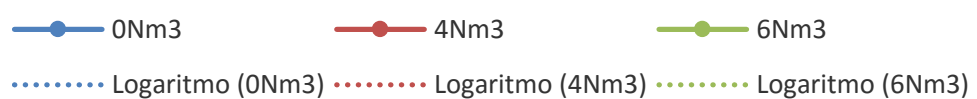

Figura 14: Curva de tendência para o valor da constante K. 
$\mathrm{Na}$ Figura 14 foi desconsiderando o bico de 3 furos, pois a penetração foi maior que $0,250 \mathrm{~m}$ tocando o fundo do convertedor. Considerando que o bico de 4 furos realmente teve uma penetração de $0,250 \mathrm{~m}$ e utilizando os outros pontos realizado a curva de tendência. Utilizando o as curvas de tendência foram estimados os valores de $\mathrm{K}$ para o bico de três furos, indicados na Tabela $\mathbf{V}$.

Tabela V: Valor estimado de $\mathrm{K}$ para diferentes vazões de ventaneiras.

\begin{tabular}{|c|c|}
\hline Vazão Ventaneira & Constante K \\
\hline $0 \mathrm{Nm3}$ & 9,59 \\
\hline $4 \mathrm{Nm} 3$ & 6,66 \\
\hline $6 \mathrm{Nm} 3$ & 5,92 \\
\hline
\end{tabular}

Com os valores da constante $\mathrm{K}$ encontrados, pode-se utilizando a equação 3.1 , escolher a melhor configuração para diferentes convertedores LD.

\section{CONCLUSÃO}

Avaliando os resultados encontrados nesse estudo, as conclusões são apresentadas a seguir.

\subsection{Sopro simples}

- Mantendo-se a vazão e DBL fixos, o aumento do número de furos no bico gera uma menor penetração do jato;

- Bico de 3 furos teve penetração que atingiu o fundo do convertedor;

- Os bicos com maior número de furos tiveram projeções mais bem distribuídas e com menor altura;

\subsection{Sopro combinado}

- Comparando o sopro combinado com o sopro simples, pode-se observar que há um aumento na penetração do jato, devido a menor densidade do banho líquido;

- A injeção de gás pelas ventaneiras diminuiu a projeção no convertedor, diminuindo o slopping do banho quando é atingido pelo jato, devido a queda da tensão superficial;

\subsection{Cálculos das constantes $\mathrm{K}$}

- A constante $\mathrm{K}$ diminui com o aumento da vazão das ventaneiras, isso ocorre como uma resposta a diminuição da densidade do banho;

- A constante $\mathrm{K}$ aumenta com a redução do número de furos do bico, devido a redução do ângulo com a vertical.

\section{Agradecimentos}

Os autores agradecem a Lumar Metals e a UFMG. 


\section{REFERÊNCIAS}

1. BARÃO, C. D., Introdução ao Refino de Aço em Convertedor a Oxigênio. In: MARTINS, A. A. R., MALYNOWSKYJ, A., SILVA, C. A., BARÃO, C. D., CASTRO, L. F. A., FARIA, M. A. A., AUAD, M. V., Fabricação de Aço em Forno Básico a Oxigênio. São Paulo: 77 Associação Brasileira de Metalurgia e Materiais,2004. Cap.1.

2. BARÃO, C. D., Sopro Combinado - Curso Fabricação de Aço em Forno Básico a Oxigênio. Vitória: Associação Brasileira de Metais, 2005.

3. BIRD, R. B., STEWART, W. E., LIGHTFOOT, E. N., Transport Phenomena. 2 ed. John Wiley \& Sons. 2002. 895p.

4. CARNeIRO, F. L. Análise Dimensional e Teoria da Semelhança e dos Modelos Físicos. Rio de Janeiro: Editora UFRJ, 1996. (1ํㅡdição 1993).

5. CASTELLAN, G. Fundamentos de Físico-Química. Tradução Cristina Maria, Pereira dos Santos, Roberto de Barros Faria. Rio de Janeiro: LTC - Livros Técnicos e Científicos Editora, 1986.

6. CASTRO, L. F. A., Fabricação de Aço em Forno Básico a Oxigênio: Termodinâmica Aplicada à Produção dos Aços em Fornos Básicos a Oxigênio. Vitória: Associação Brasileira de Metais, 2005.

7. DIAZ-CRUZ, M., MORALES, R. D., OliVARES, O., ELIAS, A. Physical and Mathematical Models of Gás-Liquid Dynamics in BOF Converters. 2002 Steelmaking Conference Proceedings. P737-748.

8. FILHO, R. C. A., FARIA, M. A. A., LOPES, H. L. P., BARBOSA, U. X., SOARES, C. Implantação do Sopro Combinado nos Convertedores da Gerdau Açominas - Processo NK-CB. Curitiba/PR, XXXIX Seminário de Aciaria (ABM), 2008.

9. GLASS, D. R., SMITH, G.C., Nozzle Theory. In: BOF Steelmaking. Volume three: Design. New York: The Iron and Steel Society of the American Institute of Mining, Metallurgical and Petroleum Engineers, Inc, 1976. Cap.8.

10. KAWAKAMI, K, Kinetics of Blowing Reaction in a Basic Oxygen Furnace, Journal of Metals, p.836-845, jul.1966.

11. Kawasaki LD-KGC Process for Combined Blowing. Kawasaki Steel Corporation, Japan, 1995.

12. LEE, C. K., NEISON, J. H., GILCHRIST, A. Correlation of the Decay Characteristics of Jets from Multi-nozzle Oxygen Lances with the Performance of the Charge in Steel Converters, Iron and Steel Internacional, p.175-184, jun.1977.

13. LIMA, H. A. A., Modelamento Físico do Sopro Combinado em Convertedores BOF: Esocla de Engenharia da UFMG, 2011. (Dissertação, Mestrado em Engenharia Metálurgica).

14. MAIA, B. T., Efeito da Configuração do Bico da Lança na Interação Jato-Banho Metálico em Convertedor LD: Escola de Engenharia da UFMG, 2007. (Dissertação, Mestrado em Engenharia Metalúrgica).

15. MAIA, B. T., BARROS, J. E. M., NASCIMENTO, L. M., GUERRA, M. S. L., TAVARES, R. P., Simulação Numérica do Sopro de Oxigênio através de Bocal Supersônico. Revista Tecnologia em Metalurgia, Materiais e Mineração v. 9, n.1, jan.- mar. 2012.

16. MAIA, B. T., Modelamento Físico e Matemático do Escoamento de Fluidos nos Processos BOF e EOF: Escola de Engenharia da UFMG, 2013. (Dissertação, Doutorado em Engenharia Metálurgica).

17. MEIDANI, A. R. N., ISAC M.,RICHARDSON, A., CAMERON, A., GUTHRIE, R. I. L., Modelling Shrouded Supersonic Jets in Metallurgical Reactor Vessels, ISIJ Internacional, v.44, n.10, p.1639, out. 2004.

18. SHAPIRO, H. N., MORAM, M. J., Fundamentals of Engineering Thermodynamics. Ed. New York: John Wiley \& Sons, 1988. P.417-435.

19.SZEKELY, J., THEMELIS, N. J., Rate Phenomena in Process Metalurgy. 1 ed. Montreal: John Wiley \& Sons, 1971. 784p. 